ISSN 1997-5902

\title{
L'apport des faibles doses d'engrais minéraux permet-il d'accroitre le rendement du maïs cultivé à forte densité ? Un exemple avec deux variétés de maïs à Lubumbashi
}

\author{
Useni Sikuzani Yannick¹, Mwema Lumbala Alain'1, Musambi Luhanga², Chinawej Mbar Mukaz \\ Dieudonné ${ }^{3}$, Nyembo Kimuni Luciens ${ }^{1}$ \\ ${ }^{1}$ Faculté des Sciences Agronomiques, Université de Lubumbashi BP 1825, Lubumbashi, RD Congo. \\ ${ }^{2}$ Faculté des Sciences Agronomiques, Université de Kamina, Kamina, RD Congo \\ ${ }^{3}$ Département des Sciences Agronomiques et Vétérinaires, Section des Sciences Exactes, Institut Supérieur \\ Pédagogique de Lubumbashi, BP 1796 Lubumbashi, RD Congo \\ Correspondance : yannickuseni@gmail.com, mwemaalain@gmail.com, luciensnyembokimuni@gmail.com
}

Original submitted in on $4^{\text {th }}$ February 2014 Published online at www.m.elewa.org on $28^{\text {th }}$ February 2014. http://dx.doi.org/10.4314/jab.v74i1.4

\section{RESUME}

Objectifs : Une expérimentation évaluant les effets de la densité de semis et de la fertilisation minérale sur le rendement du maïs, a été conduite à la ferme Kasapa de l'Université de Lubumbashi, de novembre 2012 à juin 2013.

Méthodologie et résultats : Les effets combinés de deux densités de semis (33333 plants.ha-1 et 53333 plants.ha-1) et trois doses d'engrais $(0,300 \mathrm{~kg} \mathrm{NPK}+200 \mathrm{~kg}$ urée et $400 \mathrm{~kg} N P K+800 \mathrm{~kg}$ urée) ont été étudiés sur deux variétés de maïs (PAN 53 et Unilu) dans un dispositif split split plot à 3 répétitions. Les observations ont porté sur les paramètres végétatifs, phytosanitaires et de rendement. Les doses d'engrais ont influencé la variation des tous les paramètres de croissance végétative. Le meilleur taux de levée est observé sans apport d'engrais minéraux (79\% pour UNILU et $67 \%$ pour PAN 53 ), alors que la taille des plantes augmente avec la forte dose d'engrais ( $400 \mathrm{Kg} \mathrm{NPK}+800 \mathrm{Kg}$ Urée). Parallèlement, la forte dose a augmenté la résistance à la cercosporiose et à l'helminthosporiose de la variété PAN 53. En revanche, cette variété s'est montrée sensible à la verse, alors que la variété locale résiste en l'absence de doses d'engrais minéraux. Par ailleurs, la forte dose d'engrais et la forte densité de semis ont généré des meilleurs rendements en maïs ; PAN 53 donnant des rendements nettement supérieurs (7,5 $\pm 2,9$ t.ha-1 $)$.

Conclusion et application : La faible dose d'engrais ( $300 \mathrm{Kg} \mathrm{NPK}+200 \mathrm{Kg}$ Urée) et la forte densité de semis (53333 plantes/ha) restent recommandables pour une intensification de la maïsiculture dans la zone d'étude, dans le contexte socioéconomique de cherté des engrais minéraux décriés par les agriculteurs.

Mots clés : maïs, densité de semis, dose d'engrais, variété, Lubumbashi

\section{ABSTRACT}

Can low doses of mineral fertilizers allow yield increase of maize densely grown? An example with two maize varieties in Lubumbashi

Objectives: An experiment evaluating the effects of plant density and mineral fertilization on maize yield was conducted at Kasapa farm (University of Lubumbashi), until November 2012 to June 2013. Methodology and Results: The combined effects of two plant density (33333 and 53333 plants.ha-1) and 
three fertilizer rates $(0,200 \mathrm{~kg}$ urea $+300 \mathrm{~kg}$ NPK and $800 \mathrm{~kg}$ urea $+400 \mathrm{~kg}$ NPK) were studied on two maize varieties (PAN 53 and UNILU) in a split split splot device with 3 repetitions. Observations focused on vegetative parameters, illness and yield. Doses of fertilizers have influenced the change in all vegetative parameters. The best emergence rate was observed without the addition of mineral fertilizers $(79 \%$ for UNILU and $67 \%$ for PAN 53), while plant height increases with the high dose of fertilizer (800 kg Urea + $400 \mathrm{Kg} \mathrm{NPK}$ ). Meanwhile, the high dose increased resistance to leaf spot in PAN 53 variety. However, this strain was sensitive to lodging, while the local variety resists without doses of mineral fertilizers. In addition, the high dose of fertilizer and high plant density generated higher maize yield, PAN 53 gives significantly higher yields than Unilu $\left(7.5 \pm 2.9\right.$ tha- $\left.^{-1}\right)$.

Conclusion and application: The low dose of fertilizer (300 kg NPK $+200 \mathrm{~kg}$ Urea) and high seed density (53333 plants.ha- ${ }^{-1}$ ) are recommendable for increased maize production in the study area in the socioeconomic context high cost of mineral fertilizers criticized by farmers.

KEYWORDS: maize, seeding, fertilizer dose, variety, Lubumbashi

\section{INTRODUCTION}

Le maïs (Zea mays L.) est un aliment important et une culture vivrière dans le monde. II constitue la 3ème céréale la plus cultivée au monde, après le blé et le riz. Parallèlement, sa production est la plus élevée de toutes les céréales, au niveau mondial. Originaire d'Amérique Centrale et latine, le maïs est actuellement cultivé avec succès dans les régions tempérées, tropicales et subtropicales (Malumba, 2008). En cinquante ans, la production mondiale du maïs a connu une expansion importante. Les prévisions pour les années à venir prévoient encore une nette progression de cette production, en raison notamment de la diversification des usages auquel les grains de maïs sont destinés. Avec 200 millions de tonnes produites en 1961, la production mondiale du maïs a atteint environ 712 Mt en 2005 (FAOSTAT, 2013). Jadis troisième culture vivrière après le manioc et le plantain, le maïs occupe actuellement la deuxième place parmi les cultures vivrières après le manioc en République Démocratique du Congo (RDC) (SENASEM, 2008). En RDC, le maiis est la principale céréale et elle a gagné en importance ces dernières années. A Kinshasa par exemple, la consommation annuelle $(\mathrm{kg} / \mathrm{tête}) \mathrm{de}$ maïs grains est passée de 2,84 kg en 1975 à 6,68 $\mathrm{kg}$ en 2000, soit une augmentation de $235 \%$ (Anonyme, 2009). Les rendements sont variables d'une région à une autre en $\mathrm{RDC}$, mais la moyenne nationale est de 0,8 à 1 t.ha- ${ }^{-1}$. Ces rendements sont très faibles par rapport à ceux des pays comme l'Italie (9530 kg.ha-1), le Canada
(6630 kg.ha-1), la Chine (4570 kg.ha-1) et l'Argentine (5650 kg.ha-1) (Tahir et al., 2009). Ceci est en partie due à la faible utilisation des variétés améliorées et des intrants agricoles, à l'importance des dégâts dus aux diverses pestes, et aux mauvaises pratiques culturales (SENASEM, 2008). Même si la production du maïs de la province du Katanga le place en tête des provinces de la RDC (22\% de la production nationale; SENASEM, 2008), la demande globale en 2008 était de 988 156 tonnes contre une offre de 500.854 tonnes. Le rapport offre/demande est ainsi de plus ou moins 0,5 ; ce qui crée une insécurité alimentaire à environ 9 millions d'habitants (Nyembo, 2010). Pour accroitre le rendement, il est important d'utiliser la variété améliorée et d'appliquer les bonnes pratiques culturales (Sallah et al., 2007).Deux pratiques importantes pour l'augmentation de rendement sont l'amélioration des pratiques culturales et l'apport des fertilisants (Sallah et al., 2009). Plusieurs études ont montré l'intérêt de la prise en compte de la densité de semis et de fertilisation pour accroitre la productivité de maïs (Muoneke et al., 2007 ; Sallah et al., 2009 ; Abuzar et al., 2011 ; Shafi et al., 2012). Carlone et Rinsall (1987) ont observé que les effets de la densité de semis et de la fertilisation minérale sont indépendants en culture de maïs et chaque variété requiert des densités de semis et des doses d'engrais spécifiques pour obtenir un rendement élevé. Sallah et al (1997) ont recommandé 50000plants.ha-1 et une application 
de $90 \mathrm{~kg}$ de $\mathrm{N}$ pour augmenter la production de maïs dans la savane au Ghana. Akinnifesi et al. (2007) ont reporté que l'application de $80 \mathrm{~kg} \mathrm{~N}$ par hectare était suffisante pour accroitre le rendement du maïs semé à forte densité au Malawi. Dans la ville de Lubumbashi et son Hinterland, Nyembo et al. (2012) ont montré qu'une fertilisation minérale à base de $300 \mathrm{~kg} \mathrm{NPK}+200 \mathrm{~kg}$ Urée par hectare et une densité de semis de 53333 plants par hectare seraient recommandables pour les variétés de maïs Unilu et Katanga. Les informations sur la

\section{MILIEU, MATERIEL ET METHODES}

Description du site d'étude : Cette étude a été conduite à la ferme Kasapa à Lubumbashi $\left(11^{\circ} 36^{\prime} \mathrm{S}\right.$ et $27^{\circ} 18 \mathrm{E}$, sur une altitude moyenne de $1243 \mathrm{~m}$ ) au cours de la saison culturale 2012-2013, au Sud-est de la République Démocratique du Congo. L'essai a été installé sur un bloc ayant porté une longue monoculture du maïs au cours des années antérieures. Sa flore de réponse de nouvelles variétés de maïs aux différentes doses de fertilisation minérale et densités de semis étant insuffisantes à Lubumbashi, l'objectif du présent travail est d'évaluer la réponse de deux variétés de maïs à des densités de semis et des doses des fertilisants minéraux variées. L'objectif spécifique est de déterminer la densité et la dose d'engrais à appliquer pour chaque variété en vue d'accroitre le rendement.

recolonisation est dominée par des espèces telles que Imperata cylindrica, Cynodon dactylon et Tithonia diversifolia qui occupent le terrain à des degrés de recouvrement variés. La couverture pédologique est du type ferralitique avec un pH à l'eau de 5,7 (tableau 1).

Tableau 1: Composition chimique de l'échantillon du sol du site expérimental

\begin{tabular}{|c|c|c|c|c|c|}
\hline Eléments & $\mathrm{pH}$ & Azote(\%) & Phosphore(\%) & Carbone(\%) & Potassium(\%) \\
\hline $\begin{array}{l}\text { Teneurs dans le } \\
\text { sol }\end{array}$ & 5,7 & 0,6916 & Traces & 9 & 0,0026 \\
\hline
\end{tabular}

La ferme Kasapa est caractérisée par un climat du type Cw6 suivant la classification Koppën. Elle est constituée d'une période humide d'environ 150 jours (FAO, 2005).La température moyenne est d'environ $20^{\circ} \mathrm{C}$ avec une grande stabilité interannuelle.
L'hygrométrie relative fluctue fortement au long de l'année et atteint le minimum de moins de $50 \%$ à la fin de la saison sèche (Useni et al., 2013). Les conditions climatiques qui ont prévalu au cours de la période expérimentale sont représentées dans le tableau 2.

Tableau 2. : Données climatiques de la période de l'essai

\begin{tabular}{l|lrrrrrr}
\hline Paramètres climatiques & \multicolumn{7}{c}{ Saison culturale 2012-2013 } \\
\hline Précipitations & & Décembre & Janvier & Février & Mars & Avril & Mai \\
& Quantités de pluie $(\mathrm{mm})$ & 336,8 & 324,4 & 256,6 & 174,5 & 164,5 & 0 \\
& Nombre des jours de pluies & 6 & 21 & 14 & 11 & 3 & 0 \\
& Maximum & 27,74 & 27,75 & 27,96 & 27,82 & 26,7 & 0 \\
Température $\left({ }^{\circ} \mathrm{C}\right)$ & Moyenne & 21,2 & 21,4 & 21,3 & 21,3 & 26,7 & 21 \\
& Minimum & 17,23 & 17,72 & 17,66 & 17,13 & 14,5 & 20 \\
Humidité & relative $(\%)$ & 86 & 87 & 87 & 85 & 77 & 76 \\
\hline
\end{tabular}

Source : Agence nationale de météorologie et de télédétection par satellite (METTELSAT)/station de la Luano, à Lubumbashi

Matériels : Dans cette étude, les semences de deux variétés de maïs, Unilu et PAN 53 ont été utilisées. La variété Unilu mise au point par la faculté des sciences agronomiques est la plus cultivée dans la ville. Elle est plus préférée pour sa résistance aux maladies foliaires du maïs, aux ravageurs, à la verse et son potentiel 
élevé de rendement (7 à 8,5 t.ha $^{-1}$ à une densité de 53333 plants/ha). La variété de PAN 53 produite par la firme semencière sud-africaine PANNAR a été retenue pour son rendement élevé 6 à 8 t.ha- ${ }^{-1}$ à faible densité de 33333plants/ha (Nyembo et al., 2012). En plus, PAN 53 produit 2 épis par pied. L'Urée (46\% d'azote) et le NPKS 10-20-10-6 produits par la firme OMNIA ont été utilisés comme fertilisants.

Méthodes : L'essai a été installé suivant un dispositif split split plot. Les traitements, en trois répétitions, comprenaient 2 variétés (Unilu et Pannar53), 3 doses des fertilisants inorganiques $(0,300 \mathrm{~kg} N P K+200 \mathrm{~kg}$ Urée et $400 \mathrm{~kg} N P K+800 \mathrm{~kg}$ urée) et deux densités de semis $(53333$ pieds à l'hectare et 33333 pieds par hectare). Au début de la campagne d'Octobre 2012, la parcelle a été labourée à la charrue. L'engrais minéral NPKS (10-20-10-6) a été appliqué au semis alors que l'urée a été appliquée au 30 ième pour le traitement à faible dose d'engrais et par fractionnement au $30^{\text {ème }}$ et

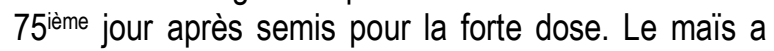

été semé à deux densités : 33333 plants/ha et 53333 plantes/ha. Trois sarclages manuels ont été effectués à 2,4 et à 10 semaines après semis. En début de la croissance le taux de levée a été déterminé par le rapport nombre de plantes levées/nombre de graines semées $x 100$. Encours de végétation, les nombres de jours à la floraison (mâle et femelle) ont été déterminés par la différence de nombre de jours entre la date de semis et la date de l'apparition des inflorescences (au moins $50 \%$ sur une parcelle); la hauteur des plantes et celle à l'insertion des épis ont été mesurées à l'aide d'un mètre ruban. A maturité, les quatre lignes de chaque traitement ont été récoltées et le rendement grain ajusté à $14 \%$ d'humidité. En revanche, le poids moyen de grains par épi, le poids de 1000 grains, et le

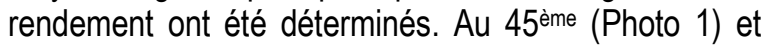
75ème jour après semis, le taux d'incidence des maladies (striure, cercosporiose et helminthosporiose) était prélevé.

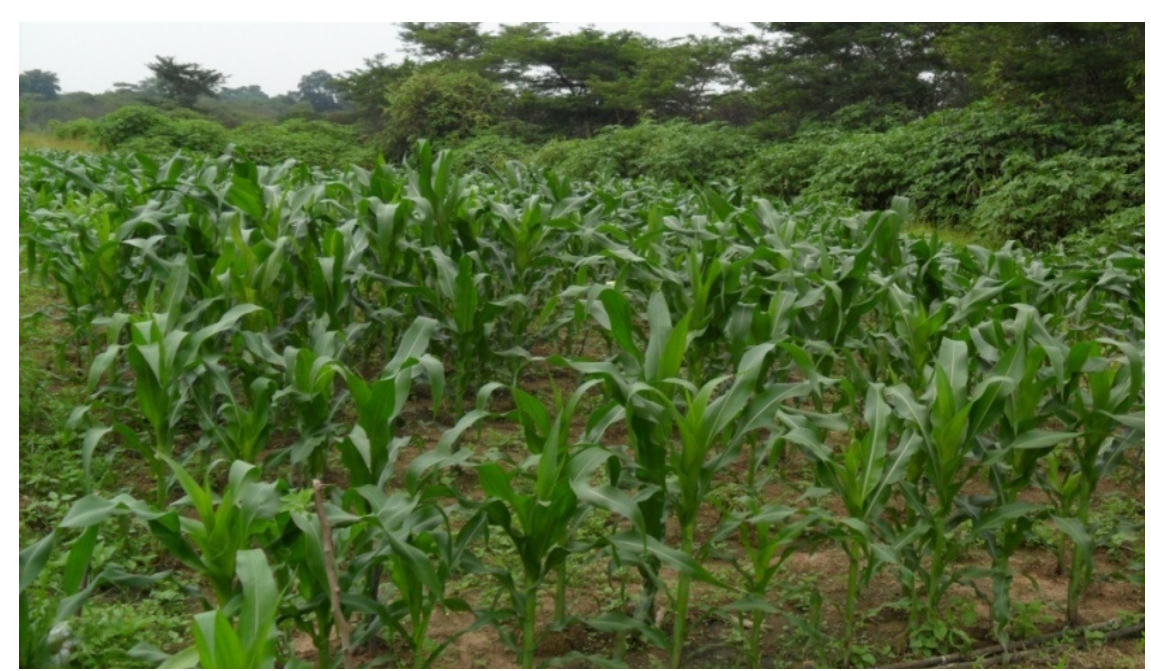

Photo 1 : Expérimentation à 45 jours après semis (Crédit : A. Mwema Lumbala et Y. Useni Sikuzani)

L'incidence était déterminée par le rapport nombre de plants malades /nombre total de plantes par parcelle $x 100$. Le taux de la résistance à la verse était calculé le jour de la récolte en prenant le nombre des pieds

\section{RESULTATS}

Effets de la fertilisation minérale et de la densité de semis sur les paramètres végétatifs de maïs (Zea mays L. var Unilu et PAN 53) à la ferme Kasapa. Les moyennes des paramètres végétatifs sont présentées dans le tableau 3. II ressort de l'analyse de variance déboutx 100/nombre total des pieds de la parcelle. L'analyse de la variance (ANOVA) avec le test de Tukey ont été utilisés pour déterminer les différences entre les traitements à l'aide du logiciel Minitab 17.

que tous les paramètres de croissance végétative observés ont subi l'influence de la dose d'engrais et des interactions qui incluent la dose, la variété et la densité de semis. 
Useni et al. J. Appl. Biosci. 2014. Des faibles doses d'engrais minéraux peuvent permettre l'augmentation du rendement du maïs cultivé densément?

Tableau 3 : Moyennes et les écarts types des valeurs des paramètres végétatifs :

\begin{tabular}{|c|c|c|c|c|c|c|c|}
\hline VARIETE & $\mathrm{DE}$ & Densité & $\begin{array}{l}\text { Taux de } \\
\text { levée }\end{array}$ & JF.M & JFF & HIE & HPL \\
\hline \multirow{6}{*}{ UNILU } & 0 & 33333 & $91,33 \pm 3,06$ & $69 \pm 1,732$ & $78,333 \pm 6,35$ & $75,33 \pm 27,02$ & $195,67 \pm 39,8$ \\
\hline & & 53333 & $93,33 \pm 3,06$ & $69 \pm 1,732$ & $78,333 \pm 6,35$ & $69 \pm 14,18$ & $192,33 \pm 27,7$ \\
\hline & D1 & 33333 & $78 \pm 9,54$ & $62,333 \pm 1,8$ & $65,667 \pm 2,2$ & $94,67 \pm 4,73$ & $242,33 \pm 7,37$ \\
\hline & & 53333 & $73,67 \pm 16,01$ & $63 \pm 2,646$ & $66,667 \pm 2,9$ & $100,33 \pm 10,6$ & $247,67 \pm 6,51$ \\
\hline & D2 & 53333 & $63,67 \pm 11,93$ & $59,667 \pm 1,8$ & $64 \pm 0,00$ & $113,33 \pm 17,1$ & $262 \pm 11,27$ \\
\hline & & 53333 & $74 \pm 14,18$ & $59,333 \pm 3,55$ & $63 \pm 2,646$ & $115,67 \pm 7,17$ & $271,33 \pm 5,51$ \\
\hline \multicolumn{2}{|c|}{ Moyenne UNILU } & & $79 \pm 14 a$ & $64 \pm 4 a$ & $69 \pm 8 a$ & $95 \pm 22 \mathrm{bs}$ & $235 \pm 36 a$ \\
\hline \multirow{6}{*}{ PAN 53} & & 33333 & $97,33 \pm 3,06$ & $69 \pm 1,732$ & $76,333 \pm 5,8$ & $97,33 \pm 5,03$ & $239,00 \pm 10,4$ \\
\hline & & 53333 & $96 \pm 2$ & $69 \pm 1,732$ & $78,333 \pm 6,31$ & $96,33 \pm 17,67$ & $233,67 \pm 30$ \\
\hline & D1 & 33333 & $55 \pm 11,36$ & $62,667 \pm 1,1$ & $66 \pm 2$ & $125,33 \pm 1,15$ & $289,67 \pm 5,51$ \\
\hline & & 53333 & $62 \pm 25,53$ & $64 \pm 0,000$ & $67,333 \pm 1,55$ & $126,67 \pm 9,29$ & $292,33 \pm 2,08$ \\
\hline & D2 & 33333 & $43,67 \pm 9,61$ & $61,667 \pm 0,57$ & $65,333 \pm 2,9$ & $114 \pm 3,51$ & $277,33 \pm 6,66$ \\
\hline & & 53333 & $48,67 \pm 11,02$ & $62 \pm 1$ & $65,333 \pm 2,9$ & $122 \pm 3,61$ & $287,00 \pm 7,94$ \\
\hline \multicolumn{2}{|c|}{ Moyenne PAN 53} & & $67 \pm 25 a$ & $65 \pm 3 a$ & $70 \pm 6 a$ & $114 \pm 15 a$ & $270 \pm 28 b$ \\
\hline \multirow{3}{*}{\multicolumn{2}{|c|}{$\begin{array}{l}\text { Moyenne doses des } \\
\text { engrais minéraux }\end{array}$}} & 0 & $94 \pm 4 a$ & $69 \pm 1 a$ & $78 \pm 5 a$ & $84 \pm 20 \mathrm{~b}$ & $215 \pm 33 b$ \\
\hline & & D1 & $67 \pm 17 b$ & $63 \pm 1 b$ & $66 \pm 2 b$ & $112 \pm 16 a$ & $268 \pm 25 a$ \\
\hline & & D2 & $58 \pm 16 b$ & $61 \pm 2 c$ & $64 \pm 2 b$ & $117 \pm 9 a$ & $275 \pm 13 a$ \\
\hline \multicolumn{2}{|c|}{ Moyenne densité } & 33333 & $71 \pm 21 a$ & $64 \pm 4 a$ & $69 \pm 7$ a & $103 \pm 20$ a & $251 \pm 35 a$ \\
\hline \multicolumn{2}{|c|}{ Effet de variété } & & 0,087 & 0,451 & 0,849 & 0,005 & 0,003 \\
\hline \multicolumn{2}{|c|}{ Effet dose d'engrais } & & 0,000 & 0,000 & 0,000 & 0,000 & 0,000 \\
\hline \multicolumn{2}{|c|}{ Effets densité } & & 0,669 & 0,803 & 0,812 & 0,832 & 0,801 \\
\hline \multicolumn{3}{|c|}{ Effets variété $\mathrm{x}$ dose } & 0,000 & 0,000 & 0,000 & 0,000 & 0,000 \\
\hline
\end{tabular}

Les différentes lettres indiquent des différences significatives et les mêmes lettres, les différences non significatives après comparaison des moyennes par le test de Tukey $(P<0,05)$. JFM : jours à la floraison male ; JFF : jours à la floraison femelle ; HIE : hauteur à l'insertion de l'épi, HPL : hauteur de la plante ; D1 $=300 \mathrm{~kg} N P K+200 \mathrm{~kg}$ Urée et D2=400kg NPK+800kg urée ; DE= doses d'engrais minéraux

Le meilleur taux de levée est obtenu sans apport d'engrais minéraux pour les deux variétés de maïs. Quant au JFM, JFF, la précocité florale est observée avec D2 pour les deux variétés. La hauteur à l'insertion de l'épi ainsi que la hauteur de plante les plus élevées sont obtenues sur la variété PAN 53. Pour les mêmes paramètres, la dose d'engrais a induit un effet significatif, de sorte que les valeurs élevées sont enregistrées D2 sur UNILU et D1 sur PAN 53. Les interactions qui induisent les fortes valeurs des paramètres sont celles qui associent les doses identifiées à la forte densité de semis, soit 53333 plantes par hectare, respectivement pour les deux variétés.

Effets de la fertilisation minérale et de la densité de semis sur l'incidence phytopathologique et la résistance à la verse : Les moyennes des valeurs obtenues pour l'incidence des maladies et la résistance à la verse sont présentées dans le tableau 4. La variété a influencé l'incidence de l'helminthosporiose 75 jours après semis. On enregistre la plus faible incidence avec la variété PAN 53. La cercosporiose a subi l'effet des doses d'engrais minéraux, de sorte qu'avec l'apport de faible dose d'engrais minéraux, l'incidence de cette maladie est nulle à 45 jours après semis. On observe également un effet combiné de dose, variété et densité sur la cercosporiose à 45 et 75 jours après semis, mais encore sur l'helminthosporiose à 75 jours après semis. La forte densité de semis permet de minimiser l'incidence de ces deux maladies sur UNILU. Par contre, l'incidence de la cercosporiose diminue avec la faible densité à 75 jours après semis. Par ailleurs, la striure à 45 et 75 jours, l'helminthosporiose à 45 jours après semis, la résistance à la verse n'ont pas été influencées ni par la variété, les doses d'engrais, ni par la densité de semis du maïs. 


\section{Useni et al. J. Appl. Biosci. 2014. Des faibles doses d'engrais minéraux peuvent permettre}

l'augmentation du rendement du maïs cultivé densément?

Tableau 4 : Les moyennes et les écarts types des valeurs des paramètres phytosanitaires (incidence maladie et résistance à la verse).

\begin{tabular}{|c|c|c|c|c|c|c|c|c|c|}
\hline VARIETE & Doses & Der & & S45 & S75 & C45 & C75 & $\mathrm{H} 75$ & R.V \\
\hline & 0 & & 33333 & $2.333 \pm 2$ & $5,33 \pm 4$ & $1.33 \pm 1$ & $71,3 \pm 21,2$ & $35,33 \pm 3,3$ & $53,2 \pm 9$ \\
\hline & & & 53333 & $1,2 \pm 2,3$ & $3,7 \pm 4$ & 0 & $68 \pm 36,2$ & $21,00 \pm 3,2$ & $52,8 \pm 15,5$ \\
\hline & D1 & & 33333 & $7 \pm 6,429$ & $9 \pm 5,1$ & 0 & $9 \pm 1$ & $26 \pm 5,1$ & $32 \pm 23,4$ \\
\hline & & & 53333 & $0,7 \pm 1,1$ & $4 \pm 3,6$ & 0 & $10 \pm 4,16$ & $16 \pm 4$ & $45,2 \pm 19,2$ \\
\hline & $\overline{\mathrm{D} 2}$ & & 33333 & $5 \pm 8,66$ & $9,7 \pm 2$ & 0 & $12,3 \pm 3,79$ & $29,33 \pm 9$ & $30,9 \pm 9$ \\
\hline UNILU & & & 53333 & $1 \pm 1,732$ & $6, \pm 2,2$ & 0 & $8,33 \pm 6,11$ & $17,33 \pm 11$ & $30,8 \pm 10,6$ \\
\hline MOYENNE & UNILU & & & $3 \pm 5 a$ & $6,2 \pm 6 a$ & $0,3 \pm 0,7 a$ & $30 \pm 33 a$ & $24,4 \pm 4 a$ & $40,8 \pm 16,53 a$ \\
\hline & 0 & & 53333 & $0,7 \pm 1,1$ & $3,3 \pm 1$ & 0 & $65 \pm 28,69$ & $21 \pm 13,23$ & $54 \pm 13,9$ \\
\hline & & & 33333 & $0,7 \pm 0,1$ & $2 \pm 2$ & 0 & $84 \pm 7,77$ & $10 \pm 5,57$ & $47,4 \pm 12,8$ \\
\hline & D1 & & 33333 & 0 & $2,6 \pm 2$ & 0 & $1 \pm 1,73$ & $5,33 \pm 4,04$ & $41,6 \pm 26,8$ \\
\hline & & & 53333 & $1,7 \pm 0,8$ & $3,7 \pm 1$ & 0 & $3,67 \pm 6,35$ & $2,33 \pm 4,04$ & $48,9 \pm 42,8$ \\
\hline & $\mathrm{D} 2$ & & 33333 & $3 \pm 2,646$ & $8 \pm 1$ & 0 & $1,67 \pm 2,89$ & $2,67 \pm 4,62$ & $39 \pm 33,8$ \\
\hline PAN 53 & & & 53333 & 0 & $7,3 \pm 1$ & 0 & $4,33 \pm 7,51$ & $4,33 \pm 3,79$ & $27,4 \pm 22$ \\
\hline MOYENNE & PAN 53 & & & $1 \pm 2 a$ & $5 \pm 6,3 a$ & $0 \pm 0 a$ & $27 \pm 37 a$ & $8 \pm 8 b$ & $43,0 \pm 24,69 a$ \\
\hline MOYENNE & & 0 & & $1,3 \pm 2 a$ & $4 \pm 3,2 \mathrm{a}$ & $0,3 \pm 1 a$ & $73 \pm 23 a$ & $22,1 \pm 16 a$ & $51,9 \pm 11,4 a$ \\
\hline DOSES D & & D1 & & $2,4 \pm 4,3 a$ & $4 \pm 4 a$ & $0 \pm 0 a$ & $6 \pm 5,2 b$ & $13 \pm 12 a$ & $41,9 \pm 25,9 a b$ \\
\hline ENGRAIS & & $\mathrm{D} 2$ & & $2,3 \pm 5 a$ & $8 \pm 9 a$ & $0 \pm 0 \mathrm{a}$ & $7 \pm 6,2 b$ & $13 \pm 13 a$ & $32 \pm 18,7 b$ \\
\hline MOYENNE & & 33333 & & $3 \pm 5 a$ & $6,3 \pm 6 a$ & $0,2 \pm 1 a$ & $27 \pm 33 a$ & $20 \pm 17 a$ & $41,8 \pm 20,4 a$ \\
\hline DENSITE & & 53333 & & $1 \pm 2 a$ & $5 \pm 6 a$ & $0 \pm 0 \mathrm{a}$ & $30,1 \pm 37 a$ & $12 \pm 10 a$ & $42,1 \pm 21,7 a$ \\
\hline Effets var & & & & 0,106 & 0,41 & 0,154 & 0,009 & 0 & 0,748 \\
\hline Effets o & s d'engr & & & 0,713 & 0,227 & 0,127 & 0 & 0,189 & 0,06 \\
\hline Effets d & & & & 0,09 & 0,381 & 0,154 & 0,779 & 0,095 & 0,964 \\
\hline Effets va & éx c & den & & 0,361 & 0,911 & 0,002 & 0 & 0,008 & 0,83 \\
\hline
\end{tabular}

Les différentes lettres indiquent des différences significatives et les mêmes lettres, les différences non significatives après comparaison des moyennes par le test de Tukey $(\mathrm{P}<0,05)$. $\mathrm{S}$ : striure ; $\mathrm{C}:$ cercosporose $; \mathrm{H}$ : Helminthosporiose ; $45: 45^{\mathrm{e} m e}$ jour $; 75$ : 75 ème jour.

Effets de la fertilisation minérale et de la densité de semis sur les paramètres de rendement de maïs (Zea mays L. var Unilu et PANNAR 53) à la ferme Kasapa. : Les moyennes de valeurs obtenues sur le poids de grains par épi, le poids de 1000 grains, le rendement en maïs grains, sont présentées dans le tableau 5. Le tableau 5 révèle que la variété a influencé le poids des grains par épi. En effet, PAN 53 présente des performances pour ce paramètre. La dose d'engrais a agi sur tous les paramètres de rendement, il ressort que les meilleurs résultats pour tous les paramètres de rendement sont obtenus avec D2 pour toutes les deux variétés. La densité de semis influence le rendement en maïs grains, en effet, le plus haut rendement est obtenu avec la densité de 53333 plants/ha pour les deux variétés. En ce qui est des effets variété $x$ doses $x$ densité, les bons résultats en PGE et PMG proviennent de D2 et 33333 plants.ha ${ }^{-1}$ pour les deux variétés, quant au rendement, il est élevé avec 53333 plants.ha- ${ }^{-1}$ et $400 \mathrm{Kg}$ de NPK et $800 \mathrm{Kg}$ d'urée. 
Tableau 5 :. Les moyennes et les écarts types des valeurs des paramètres de rendement.

\begin{tabular}{|c|c|c|c|c|c|}
\hline VARIETE & Doses & Densité & PGE (g) & PMG (g) & RDT (t.ha-1) \\
\hline \multirow{6}{*}{ UNILU } & \multirow[t]{2}{*}{0} & 33333 & $110,5 \pm 36,4$ & $232,7 \pm 15,6$ & $3,68 \pm 1,21$ \\
\hline & & 53333 & $95,2 \pm 28,4$ & $206,5 \pm 15,4$ & $5,08 \pm 1,45$ \\
\hline & \multirow[t]{2}{*}{ D1 } & 33333 & $155,9 \pm 21,8$ & $271,5 \pm 23,8$ & $5,2 \pm 0,73$ \\
\hline & & 53333 & $149,9 \pm 15,7$ & $273,6 \pm 15,2$ & $7,99 \pm 0,84$ \\
\hline & \multirow[t]{2}{*}{$\mathrm{D} 2$} & 3333 & $175,5 \pm 11,7$ & $323,7 \pm 27,9$ & $5,85 \pm 0,4$ \\
\hline & & 53333 & $170,3 \pm 4,1$ & $304,8 \pm 20,2$ & $9,09 \pm 0,22$ \\
\hline \multicolumn{2}{|c|}{ MOYENNE UNILU } & & $142,9 \pm 36 b$ & $268,7 \pm 44,4 \mathrm{a}$ & $6,1 \pm 2,1 \mathrm{a}$ \\
\hline & & 3333 & $128,2 \pm 24,6$ & $259,4 \pm 10,2$ & $4,27 \pm 0,82$ \\
\hline & & 53333 & $99 \pm 27,2$ & $206,5 \pm 15,4$ & $5,28 \pm 1,45$ \\
\hline & \multirow[t]{2}{*}{ D1 } & 33333 & $201,9 \pm 27,2$ & $307,6 \pm 7,1$ & $6,73 \pm 0,91$ \\
\hline & & 53333 & $196,6 \pm 23,8$ & $302,7 \pm 14,6$ & $10,49 \pm 1,27$ \\
\hline & D2 & 33333 & $198,2 \pm 21,3$ & $324,2 \pm 15,9$ & $6,61 \pm 0,71$ \\
\hline \multicolumn{2}{|l|}{ PAN 53} & 53333 & $217,2 \pm 11,1$ & $322,1 \pm 22,1$ & $11,58 \pm 0,59$ \\
\hline \multicolumn{2}{|c|}{ MOYENNE PAN 53} & & $173,5 \pm 49 a$ & $291 \pm 38,5$ a & $7,5 \pm 2,9 a$ \\
\hline \multirow{3}{*}{\multicolumn{2}{|c|}{$\begin{array}{l}\text { MOYENNES DOSES DES } \\
\text { ENGRAIS MIN }\end{array}$}} & 0 & $108,2 \pm 28,5 b$ & $232 \pm 24,7 \mathrm{c}$ & $4,6 \pm 1,3 b$ \\
\hline & & D1 & $176 \pm 31 a$ & $288,7 \pm 22,2 b$ & $7,6 \pm 2 a$ \\
\hline & & D2 & $190,3 \pm 22,7 \mathrm{a}$ & $318,7 \pm 20,5 \mathrm{a}$ & $8,3 \pm 2,4 a$ \\
\hline \multirow{2}{*}{\multicolumn{2}{|c|}{ MOYENNES DENSITE }} & 33333 & $161,7 \pm 40,8 a$ & $286,4 \pm 38,6$ a & $5,39 \pm 1,36 b$ \\
\hline & & 53333 & $154,7 \pm 50,1 a$ & $273,3 \pm 46,2 \mathrm{a}$ & $8,25 \pm 2,67$ a \\
\hline \multirow{2}{*}{\multicolumn{2}{|c|}{$\begin{array}{l}\text { Effet variété } \\
\text { Effet doses } d^{\prime}\end{array}$}} & & 0,040 & 0,117 & 0,114 \\
\hline & ais & & 0,000 & 0,000 & 0,000 \\
\hline \multirow{2}{*}{\multicolumn{2}{|c|}{$\begin{array}{l}\text { Effet densité } \\
\text { Effet variété } x\end{array}$}} & & 0,650 & 0,360 & 0,000 \\
\hline & & & 0,000 & 0,000 & 0,000 \\
\hline
\end{tabular}

Les différentes lettres indiquent des différences significatives et les mêmes lettres, les différences non significatives après comparaison des moyennes par le test de Tukey $(P<0,05)$.

\section{DISCUSSION}

Les résultats obtenus sur les effets de la densité de semis indiquent de différence non significative pour les paramètres végétatifs et phytosanitaires, des différences significatives étant obtenues pour les paramètres de rendement. En dépit de différence non significative décelée, les résultats indiquent tout de même que la taille de plante était la plus élevée sur les parcelles à forte densité de semis. Abuzar et al. (2011) évaluant les effets de la densité de semis sur la production du maïs ont montré que l'indice de la surface foliaire (LAl), influencé par le génotype, la densité de peuplement, le climat et la fertilité du sol, augmente à forte densité. En outre, il se produit une croissance en hauteur de la plante consécutive à l'augmentation de l'activité photosynthétique et de la compétition entre les individus. Les études antérieures de Saberali (2007) ont montré une augmentation de LAl et de la biomasse sous une forte densité de semis. Sangakkara et al. (2004) justifie cela par le fait que l'augmentation de la densité de semis entraine une forte compétition pour le prélèvement de nutriment d'une part et de l'autre une compétition pour l'interception de la lumière. Hassan (2000) a montré que la taille des plantes augmente consécutivement à la densité de semis, de 47600 à 71400 plants.ha $^{-1}$. Le rendement obtenu avec le peuplement de 53333 plants. ha-1 était plus élevé que celui obtenu avec 33333 plants. ha-1 ${ }^{-1}$. Ceci se justifie par le fait qu'en dépit de la compétition entre les plantes sur les parcelles à fortes densités (Zamir et al., 2011) et de la compétition en nutriment (Sangakkara et al., 2004), le rendement obtenu est la somme des productions individuelles. Le rendement élevé obtenu sur les parcelles à forte densité se justifierait ainsi par l'optimisation de l'utilisation du sol et des ressources environnementales par la culture. Eman (2001) estime que le rendement élevé du maïs sur les parcelles à forte densité est généralement dû au nombre élevé d'épis par ligne et au nombre élevé de rangées de graines par épi. En outre, Nyembo (2010) justifie cette augmentation par le fait que la densité de semis est aussi une composante clé du rendement, étant donné que le rendement obtenu est la somme des productions individuelles. Hassan (1987) et Muoneke et al (2007) ont indiqué que 


\section{Useni et al. J. Appl. Biosci. 2014. Des faibles doses d'engrais minéraux peuvent permettre}

l'augmentation du rendement du maïs cultivé densément?

la densité de semis affecte moins le cycle végétatif du maïs. D'autres études ont montré ainsi que la densité de plantation est un facteur clé pour l'atteinte d'un rendement élevé (Esechie, 1992 ; Akbar et al., 2002 ; Mojtaba et al., 2012). Des résultats similaires ont été obtenus par Maradapour et al. (2013) dans une étude orientée vers l'évaluation des effets de la date et de la densité de semis sur le rendement du riz en Iran. Pour ces auteurs, l'augmentation de rendement du riz est associée à celle de la densité de semis et le rendement le plus élevé (6403 kg.ha-1) a été obtenu à la forte densité de 68 plants $/ \mathrm{m}^{2}$.

La comparaison des variétés entre elles indiquent que la variété PAN 53 présente une taille élevée en même temps qu'un rendement élevé contrairement à la variété Unilu. Par contre, les plantes de la variété Unilu, à taille réduite résistent mieux à la verse que celles de la variété PAN 53. Plusieurs travaux conduits dans la ville de Lubumbashi ont montré la supériorité de la variété de maïs PAN 53 par rapport à la variété Unilu quant à la dose de la fertilisation minérale et au fractionnement de l'azote (Chukiyabo, 2012 ; Lumbu, 2012 ; Mbaya, 2012 ; Nyembo et al., 2013). Pour l'incidence de 3 maladies foliaires, les résultats obtenus ont montré que des niveaux d'incidence similaires ont été obtenus entre les variétés, les doses d'engrais et les deux densités de semis. Des résultats similaires ont été obtenus par Chukiyabo (2012) ; Lumbu (2012) et Mbaya (2012) dans les conditions pédoclimatiques de Lubumbashi, montrant ainsi l'intérêt de l'amélioration génétique du maïs dans lutte contre les 3 maladies foliaires rependues dans la région (Nyembo, 2010).

Pour les doses d'engrais appliquées, les résultats montrent que le taux de levée diminue avec l'apport d'engrais. Des résultats similaires ont été évoqués en conditions de semis au cours des périodes de stress hydrique par Nyembo et al. (2012) sur le maïs à Lubumbashi et Gala et al. (2011) sur le riz en Côte d'Ivoire. Par contre, la croissance en hauteur et la précocité sont associées à l'apport des fertilisants, notamment l'azote contenu dans les engrais minéraux (Gala et al., 2011 ; Nyembo et al., 2012). Toutefois, des

\section{CONCLUSION}

L'utilisation des 3 doses des engrais minéraux ( 0 , $300 \mathrm{~kg} \mathrm{NPK}+200 \mathrm{~kg}$ urée et $400 \mathrm{~kg}$ NPK $+800 \mathrm{~kg}$ urée), 2 densités de semis (33333 et 53333 plants.ha-1) et 2 variétés améliorées de maïs (Unilu et Pan53), a été explorée en vue de contribuer à la sécurité alimentaire des populations de Lubumbashi par l'intensification de la maïsiculture. Les résultats obtenus ont montré que la effets similaires sont obtenus avec D1 et D2. Ceci est dû à l'excès des apports fertilisants, étant donné qu'il existe un seuil d'application dans tout apport de fertilisants (Useni et al., 2012). En effet, Lafond (2004) indique à titre d'exemple que les apports d'azote élevés peuvent aussi provoquer la verse chez le maïs et ainsi réduire les rendements en grains. L'apport des engrais chimiques a accru de manière significative le rendement du maïs comparativement aux parcelles témoins pour lesquels l'acidité, la déficience en nutriments et l'absence d'apport organique, caractéristiques des sols acides de Lubumbashi (Kasongo et al., 2013) ont induit une chute drastique des rendements. Les résultats obtenus, montrant une augmentation significative des rendements avec l'apport des engrais minéraux, montre l'intérêt de la fertilisation minérale dans l'intensification de la maïsiculture. Pypers et al. (2010) ont trouvé que les engrais augmentent 40 à $100 \%$ de rendement de culture dans les sols Kalongo et Civu, dans le Sud Kivu, en République Démocratique du Congo. Nyembo et al. (2012) évaluant les effets de doses variées des engrais minéraux sur le rendement du maïs à Lubumbashi avec 5 doses d'engrais minéraux( $\mathrm{D} 0=$ témoin sans fertilisation; D1 $=300 \mathrm{Kg} \mathrm{NPKS}+200 \mathrm{Kg}$ Urée; D2 $=400 \mathrm{Kg} \mathrm{NPKS}+800 \mathrm{Kg}$ Urée; D3 $=200 \mathrm{Kg}$ NPKS+400 Kg Urée; D4=500Kg NPKS+ $600 \mathrm{Kg}$ Urée), ont montré que le rendement faible de 2,7 tha- ${ }^{-1}$ a été obtenu sur le témoin ; alors que c'est le triple de D0 qui a été obtenu sur D1, le quadruple sur D2 et D3; et le quintuple sur D4. Quant aux interactions, les résultats obtenus ont montré que, quelle que soit la variété, les rendements élevés sont obtenus avec la forte densité de semis couplée à la faible dose d'engrais. Des résultats similaires ont été obtenus par Sallah et al. (2009). Ces auteurs, évaluant les effets de 3 densités de semis $\left(30000,50000\right.$ and 70000 plants ha- $\left.^{-1}\right)$ et 3 niveaux de fertilisation azotée $\left(0,80\right.$ et $\left.160 \mathrm{~kg}^{-h} \mathrm{~h}^{-1}\right)$ au Rwanda, ont montré que le rendement le plus élevé était obtenu à la densité moyenne couplée à la faible dose de fertilisation azotée

densité de semis n'a pas affecté les paramètres étudiés, hormis le rendement; alors que de différence significatives ont été révélées entre les doses d'engrais minéraux et les variétés quant au rendement obtenu. En effet, les rendements élevés et similaires ont été obtenus sur D1 et D2 alors que PAN 53 est la plus productive que Unilu. Ainsi, l'augmentation de la 


\section{Useni et al. J. Appl. Biosci. 2014. Des faibles doses d'engrais minéraux peuvent permettre}

l'augmentation du rendement du maïs cultivé densément?

densité de semis et la réduction de la dose des engrais minéraux constituent un moyen approprié d'augmentation de la production du maïs à Lubumbashi. Cette étude constitue une un apport à

\section{REFERENCES}

Abuzar MR, Sadozai GU, Baloch MS, Baloch AA, Shah $\mathrm{IH}$, Javaid T, Hussain N, 2011. Effect of plant population densities on yield of maize. The Journal of Animal \& Plant Sciences, 21(4): 692-695

Akbar H, Miftahullah MT, Jan A, Ihsanullah A, 2002. Yield potential of sweet corn as influenced by different levels of nitrogen and plant population. Asian J. Plant Sci., 1: 631-633.

Akinnifesi FK, Makumba W, Sileshi G, Ajayi OC, Mweta $D, 2007$. Synergistic effect of inorganic $N$ and $P$ fertilizers and organic inputs from Gliricidia sepium on productivity of intercropped maize in Southern Malawi. Plant and Soil 294 (1-2): 203-217.

Anonyme, 2009. Étude du secteur agricole : Rapport préliminaire, bilan-diagnostic et notes d'orientation. Ministère national de l'agriculture pêche et élevage, Tecsul et GECT, Kinshasa, RDC. $337 \mathrm{p}$

Carlone MR, Russell WA, 1987. Response to plant densities and nitrogen levels for four maize cultivars from different eras of breeding. Crop Science 27: 465-370.

Chukiyabo KM, 2012. Evaluation et sélection de nouvelles variétés de maïs en provenance de PANNAR dans les conditions pédoclimatiques de Lubumbashi. Mémoire de fin d'étude, Faculté des Sciences Agronomiques, Université de Lubumbashi, $50 \mathrm{p}$

Emam $Y, 2001$. Sensitivity of grain yield components toplant population density in non-prolific maize (Zea mays) hybrids. Indian J. Agric. Sci. 71(6):367-370.

Esechie HA, 1992. Effect of planting density on growth and yield of irrigated maize in the Batinah coast region of Oman. J. Agri. Sci., 119: 165169.

FAO/STAT(2013).http://faostat.fao.org/default.aspx?ali as=faostat\&lang=fr (consulté le 26/11/2013)

Galla TJ, Camara M, Yao Kouame A, Keli ZJ, 2011. Rentabilité des engrais minéraux en riziculture de plateau : cas de la zone de Gagnoa dans le centre ouest de la Cote d'Ivoire. Journal of applied bioscience 46: 3153-3162 l'amélioration des pratiques culturales en maïsiculture, dans un contexte où la cherté des engrais minéraux et de la semence des variétés améliorées est très souvent un frein à l'intensification de la maïsiculture.

Hassan AA, 2000. Effect of plant population density on yield and yield components of 8 Egyptian maize hybrids. Bull. Faculty Agric. Uni. Cairo., 51: 1-16.

Hassan AE, 1987. Performance of three corn cultivars (Zea mays L.) as affected by plant density in Riyadh region. J. Agric. Bio. Sci., 5: 199-203.

Kasongo LME, Mwamba MT, Tshipoya MP, Mukalay MJ, Useni SY, Mazinga KM, Nyembo KL, 2013. Réponse de la culture de soja (Glycine max L. (Merril) à l'apport des biomasses vertes de Tithonia diversifolia (Hemsley) A. Gray comme fumure organique sur un Ferralsol à Lubumbashi, R.D. Congo. Journal of Applied Biosciences 63: 4727 - 4735

Lafond J, 2004. Fractionnement de la fertilisation azotée minérale et organique: Effet sur la productivité du canola de printemps et sur les nitrates du sol. Canadian Journal of Soil Science 84: 491-501.

Lumbu BL, 2012. Effets des doses croissantes des fertilisants minéraux (NPK et Urée) sur la production de deux variétés de maïs (Zea mays L. var Unilu et Pan 53) à Lubumbashi. Mémoire de fin d'étude, Faculté des Sciences Agronomiques, Université de Lubumbashi, 52p

Malumba KP, 2008. Influence de la température lors du séchage sur les propriétés technofonctionnelles du maïs. Thèse de doctorat, Gembloux Faculté Universitaire des Sciences agronomiques, 212p.

Mbaya NGJ, 2012. Effets combinés du fractionnement de l'urée et de la variété sur le rendement du maïs à la ferme Kasapa, Lubumbashi. Mémoire de fin d'étude, Faculté des Sciences Agronomiques, Université de Lubumbashi, 49p

Mojtaba NKK, Saied KK, Taheri G, 2012. Effects of plant density and variety on some of morphological traits, yield and yield components of baby corn (Zea mays L.). International Research Journal of Applied and Basic Sciences 3 (10): 2009-2014

Moradpour S, Roja K, Mehrie B, Meysam GK, 2013.Effect of planting date and planting density on rice yield and growth analysis (Fajr 
variety). International Journal of Agriculture and Crop Sciences 5 (3): 267-272

Muoneke CO, Ogwuche MAO, Bakul BA, 2007. Effect of maize planting density on the performance of maize/soybean intercropping system in a savannah agroecosystem. African Journal of Agricultural Research 2(12): 667-677

Nyembo KL, 2010. Augmentation du rendement du maïs par l'exploitation de l'effet hétérosis des hybrides produits au Katanga, République Démocratique du Congo. Thèse de doctorat, Faculté des sciences agronomiques, Université de Lubumbashi, 157p.

Nyembo KL, Useni SY, Mpundu MM, Bugeme MD, Kasongo LE, Baboy LL, 2012. Effets des apports des doses variées de fertilisants inorganiques (NPKS et Urée) sur le rendement et la rentabilité économique de nouvelles variétés de Zea mays L. à Lubumbashi, SudEst de la RD Congo. Journal of Applied Biosciences 59: 4286- 4296

Pypers $P$, Vandamme E, Sanginga JM, Tshibinda T, Walangululu MJ, Merckx R, Vanlauwe B, 2010. $\mathrm{K}$ and $\mathrm{Mg}$ deficiencies corroborate farmer's knowledge of soil fertility in the Highland of South-Kivu, Democratic Republic of Congo. in E.M. Bagura (Ed): évaluation de l'efficacité d'usage des engrais dans les sols dégradés du Sud-Kivu sur la culture du maïs et du haricot commun: cas du groupement de burhale". Mémoire de fin d'études, Université Evangelique en Afrique, (2010) 59p

Saberali SF, 2007. Influence of plant density and planting pattern of corn on its growth and yield under competition with common Lambesquarters (Chenopodium album L.). Pajouhesh and Sazandegi. 74: 143-152.

Sallah PYK, Mukakalisa S, Nyombayire A, Mutanyagwa $P, 2009$. Response of two maize varieties to density and nitrogen fertilizer in the highland zone of Rwanda. Journal of Applied Biosciences 20: 1194-1202

Sallah PYK, Njeru RW, Akinyemi SOS, Nyirigira A, Senkesha N, Gashabuka E, Kabayiza E, Nyombayire A, 2007. Diagnostic survey of the farming systems in Rusogo watershed in Nyabihu District of Rwanda: Cropping systems and challenges. In: Njeru RW, Kagabo DM, Ndabamenye $T$, Kayiranga $D$, Ragama $P$, Sallah PYK, Nkerabahizi D, Ndayiragije A, Ndiramiye L, Night G, Akinyemi SOS \&
Kanuya N (eds.) Proceedings, Sustainable agricultural productivity for improved food security and livelihoods, Kigali, Rwanda, March 26-28, 2007. pp. 82-91.

Sallah PYK, Twumasi-Afriyie S, Frimpong-Manso PP, 1997. Studies on performance of some openpollinated maize cultivars in the Guinea savanna. I. Effects of plant density, nitrogen level and their interactions on yield. Ghana Journal of Agricultural Science 30:151-159.

Sangakkara UR, Bandaranayake PSRD, Gajanayake JN, Stamp P, 2004. Plant populations and yield of rainfed maize grown inwet and dry seasons of the tropics. Maydica. 49:83-88.

SENASEM, 2008. Catalogue variétal des cultures vivrières : Céréales (maïs, riz), Légumineuses (haricot, soja, niébé), Plantes à tubercules (manioc, patate douce, pomme de terre), Bananier. Appui du projet CTB/MINAGRI, Kinshasa, p153.

Shafi M, Jehan B, Sajjad A, Hamayoon K, Mohammad AK, Mohammad S, 2012. Effect of planting density on phenology, growth and yield of maize (Zea mays L.). Pak. J. Bot., 44(2): 691696, 2012.

Tahir M, Javed MR, Tanveer A, Nadeem MA, Wasaya A, Bukhari SAH, Rehman JU, 2009. Effect of different herbicides on weeds, growth and yield of spring planted maize (Zea mays L.). Pak. J. Life Soc. Sci. 7(2): 168-174.

Useni SY, Chukiyabo KM, Tshomba KJ, Muyambo ME, Kapalanga KP, Ntumba NF, Kasangij A-KP, Kyungu K., Baboy L.L., Nyembo K.L., Mpundu M.M., 2013. Utilisation des déchets humains recyclés pour l'augmentation de la production du maïs (Zea mays L.) sur un ferralsol du sudest de la RD Congo. Journal of Applied Biosciences 66:5070 - 5081

Zamir MSI, Ahmad AH, Javeed HMR, Latif T, 2011. Growth and yield behaviour of two maize hybrids (Zea mays L.) towards different plant spacing. Cercetări Agronomice în Moldova. 14(2): 33-40. 